\title{
Optimization of ambient intelligence systems
}

\section{Tin-Chih Toly Chen ${ }^{1} \cdot$ W. Art Chaovalitwongse ${ }^{2} \cdot$ I-Hsuan Hong ${ }^{3}$}

Published online: 1 September 2018

(c) Springer-Verlag GmbH Germany, part of Springer Nature 2018

Ambient intelligence (AmI) is a future vision in which an environment supports the people inhabiting it in an unobtrusive, interconnected, adaptable, dynamic, embedded, and intelligent way. In this vision, an environment is sensitive to the needs of its inhabitants and capable of anticipating their needs and behavior. There are various forms of AmI systems including smart home, smart factories, smart shops, mobile guides, virtual tours, ubiquitous health care systems, online social networks, among others.

Optimizing an AmI system is a controversial problem. Numerous AmI systems involve human decision-making processes, such as deciding whether to follow the results of an online restaurant recommendation system. However, human decisionmaking is not strictly optimizing in an economical and mathematical sense. In addition, representing people's subjective feelings by using a simple scale, as performed in several other fields, is inappropriate. Therefore, an AmI system optimization problem cannot be resolved simply by applying heuristics.

Optimizing an AmI system is also a difficult task. First, bulk information may need to be processed, which renders the optimization model extremely large. In addition, such data are dynamic and often incomplete, and this phenomenon poses a challenge to the adaptability and robustness of the optimization model. Furthermore, users' preferences for the recommended service are unclear, vague, inconsistent, and difficult to quantify. Setting a single objective function that is applicable to everyone is thus a difficult task. In addition, cultural differences also considerably influence optimizing an AmI system. This implies that the relationship among the variables

Tin-Chih Toly Chen

tolychen@ms37.hinet.net

W. Art Chaovalitwongse

artchao@uark.edu

I-Hsuan Hong

ihong@ntu.edu.tw

1 Department of Industrial Engineering and Management, National Chiao Tung University, Hsinchu City, Taiwan

2 College of Engineering, University of Arkansas, Fayetteville, USA

3 Institute of Industrial Engineering, National Taiwan University, Taipei, Taiwan 
in the optimization model may differ according to culture. Furthermore, data incompleteness is another problem. In most cases, users are unwilling or find it inconvenient to answer all the questions, for example, when in need of a distant emergency care. However, the system must still assist the user by making decisions based on incomplete information.

In most AmI systems, only a few (or countable) alternatives are available, thus forming a discrete feasible region with limited solutions. Most problems of optimizing AmI systems have been formulated as mixed integer-linear or integer-nonlinear programming problems. This special issue is intended to provide technical details of the optimization of AmI systems and the corresponding applications. These details will hold great interest for researchers in ambient intelligence, optimization, system science, operations research, information management, artificial intelligence, and computational intelligence, as well as for practicing managers and engineers. This special issue features a balance between state-of-the-art research and practical applications. This special issue also provides a forum for researchers and practitioners to review and disseminate quality research work on optimizing AmI systems and the critical issues for further development. After a strict review process, seven articles from researchers around the world were accepted.

A smart hospital can react to emergencies and unexpected events in real time. However, emergencies disrupt the established schedules of operating rooms in a smart hospital. This problem needs to be addressed, so as to save as many lives as possible. To this end, in the first paper, Al-Refaie et al. formulated three optimization models to optimize the schedules of operating rooms by considering unexpected events, thereby incorporating the surgeries of emergent patients into the established schedules.

Job sequencing and scheduling has been considered as one of the basic intelligences of a smart factory. In the second paper, Lin solved two sequencing and scheduling problems in a factory with correlated parallel machines: one minimizes the makespan; the other considers the release times in minimizing the total weighted tardiness. Both problems are theoretically NP-hard. In addition, a variety of levels and combinations of machine correlations and job correlations in the processing times are taken into account. Lin formulated and optimized the mathematical programming models of the two problems. A branch-and-bound algorithm was also proposed to facilitate the solution finding.

In the third paper, Lin et al. tried to resolve a problem in mobile hotel recommendation-some users chose dominated hotels instead of the recommended hotels. This problem is difficult to resolve because there is no reason to recommend a hotel that is inferior to another in all aspects. To address this problem, they added an artificial dimension to each hotel to model unknown personal preferences. The weights assigned to all of the dimensions were derived by solving an integer-nonlinear programming (INLP) problem aimed at maximizing the successful recommendation rate for the historical data. The results of a regional experiment supported the effectiveness of the proposed methodology.

Operating rooms (ORs), one of the most crucial hospital resources that generate the highest costs, are prone to bottlenecking. In the fourth paper, Al-Refaie et al. tried to optimize the multiple-period scheduling of patients in the ORs and intensive 
care units (ICUs) of a hospital. To this end, two mathematical programming models were proposed and solved to determine the start times and sequences of patient surgeries, start times of the recovery procedures, and ICU bed assignments. For the ORs, the total cost of hospitalization, overtime, undertime, and cancelation was minimized while considering patient prioritization and satisfaction. For the ICUs, the total overtime cost was minimized.

To increase the ecological sustainability of manufacturing, enhancing the yield of each product is a critical task that eliminates waste and increases profitability. An equally crucial task is to estimate the future yield of each product so that the majority of factory capacity can be allocated to products that are expected to have higher yields. To this end, Chen and Wang proposed a fuzzy collaborative intelligence (FCI) approach. In the FCI approach, each expert constructs an artificial neural work (ANN) to fit an uncertain yield learning process for estimating the future yield with a fuzzy value. Then, fuzzy intersection is applied to aggregate the fuzzy yield estimates from different experts. According to the experimental results, the proposed methodology outperformed five existing methods in improving the estimation accuracy.

Most smart systems are automated operation systems. The monitoring and checking of such systems is a critical task to make judgments and provide solutions. To fulfill this task, Liu et al. developed an intelligent fuzzy control system to evaluate and improve the performance of a supervisor. In addition, an alarm signal was generated by the intelligent fuzzy control system to remind the supervisor. The experimental results showed that the effect of the intelligent fuzzy control system on improving the supervisory performance was significant.

Factory simulation is another intelligence of a smart factory. However, the large amount of money, time, efforts, and know-how required for conducting a factory simulation study force a factory to pursue the persistent application of the factory simulation model, i.e. the sustainability of the factory simulation model. In the last paper, Chen et al. proposed a multi-granularity approach to estimate the sustainability of a factory simulation model based on short-term evidences. According to the results of a simulation experiment, the multi-granularity approach reduced the input space by $89 \%$ and maintained a very high estimation accuracy.

I would like to thank Operational Research: An International Journal Editor-inChief, Nikolaos Matsatsinis, for fully supporting the release of this special issue. I am also grateful to the contributors who shared their research as well as to the reviewers who spared their valuable time to review papers. I would also like to thank the journal's staff. Without their support and professional assistance, prepublication would not have been possible. 